\title{
Inherent promoter bidirectionality facilitates maintenance of sequence integrity and transcription of parasitic DNA in mammalian genomes
}

\author{
Paul Kalitsis*1,3 and Richard Saffery 2,3
}

Address: ${ }^{1}$ Chromosome and Chromatin Research, Murdoch Childrens Research Institute, Royal Children's Hospital, Parkville, Melbourne 3052 , Victoria, Australia, 2 Developmental Epigenetics, Murdoch Childrens Research Institute, Royal Children's Hospital, Parkville, Melbourne 3052, Victoria, Australia and ${ }^{3}$ Department of Paediatrics, University of Melbourne, Parkville, Melbourne 3052, Victoria, Australia

Email: Paul Kalitsis* - paul.kalitsis@mcri.edu.au; Richard Saffery - richard.saffery@mcri.edu.au

* Corresponding author

Published: 27 October 2009

BMC Genomics 2009, 10:498 doi:10.1186/147/-2164-10-498
Received: 16 January 2009

Accepted: 27 October 2009

This article is available from: http://www.biomedcentral.com/I47I-2/64/I0/498

(C) 2009 Kalitsis and Saffery; licensee BioMed Central Ltd.

This is an Open Access article distributed under the terms of the Creative Commons Attribution License (http://creativecommons.org/licenses/by/2.0), which permits unrestricted use, distribution, and reproduction in any medium, provided the original work is properly cited.

\begin{abstract}
Background: Many mammalian genes are arranged in a bidirectional manner, sharing a common promoter and regulatory elements. This is especially true for promoters containing a $C_{p} G$ island, usually unmethylated and associated with an 'open' or accessible chromatin structure. In evolutionary terms, a primary function of genomic methylation is postulated to entail protection of the host genome from the disruption associated with activity of parasitic or transposable elements. These are usually epigenetically silenced following insertion into mammalian genomes, becoming sequence degenerate over time. Despite this, it is clear that many transposable element-derived DNAs have evaded host-mediated epigenetic silencing to remain expressed (domesticated) in mammalian genomes, several of which have demonstrated essential roles during mammalian development.
\end{abstract}

Results: The current study provides evidence that many CPG island-associated promoters associated with single genes exhibit inherent bidirectionality, facilitating "hijack" by transposable elements to create novel antisense 'head-to-head' bidirectional gene pairs in the genome that facilitates escape from host-mediated epigenetic silencing. This is often associated with an increase in $C_{P G}$ island length and transcriptional activity in the antisense direction. From a list of over 60 predicted protein-coding genes derived from transposable elements in the human genome and $\mathbf{4 0}$ in the mouse, we have found that a significant proportion are orientated in a bidirectional manner with $C_{P G}$ associated regulatory regions.

Conclusion: These data strongly suggest that the selective force that shields endogenous $C_{p} G$ containing promoter from epigenetic silencing can extend to exogenous foreign DNA elements inserted in close proximity in the antisense orientation, with resulting transcription and maintenance of sequence integrity of such elements in the host genome. Over time, this may result in "domestication" of such elements to provide novel cellular and developmental functions.

\section{Background}

The emergence of novel gene functions is an essential driving force behind the evolution of species. Many molecular mechanisms have been described that contribute to this process including gene duplication, exon shuffling, retroposition, transposable element insertion, 
lateral (horizontal) gene transfer, and gene fusion/fission events [1].

One of these mechanisms, transposable or mobile elements, are segments of DNA encoding genes that assist in DNA excision, replication and integration of the elements into new regions of the genome. Until recently transposable elements (TEs) have been considered parasitic or selfish DNAs that contribute little to the host organism $[2,3]$. These elements generally exist as neutrally evolving inactive DNA remnants that are epigenetically silenced by the host genome to prevent transcription and subsequent transposition $[4,5]$. Such elements are therefore subject to little selective pressure and subsequently acquire sequence variation (mutations) over time. However, it has recently been shown that some TEs escape host cell silencing to become 'domesticated' by host genomes resulting in the formation of novel genes [6-8]. Such domesticated elements are involved in many cellular and developmental functions including placental development, viral resistance, chromatin structure, DNA recombination and repair, gene regulation, apoptosis and brain development [8]. Given these facts, it appears that a complex evolutionary interplay exists between genomic silencing of transposon elements to prevent their proliferation, and co-option of transposon-encoded proteins to provide novel cellular functions in higher eukaryotic genomes.

In an attempt to understand and identify the molecular mechanism/s of domestication we have examined the number of such genes in the human and mouse genomes and analysed the features of their genomic insertions sites. We classified each according to gene/promoter structure, degree of conservation, expression profile, and transposable element type.

Whereas previous studies have suggested that the majority of transposable element domestication arises via a gene fusion event or genomic insertion in close proximity to a cryptic promoter $[1,8,9]$, our analysis has revealed that only a small proportion of co-opted TE-derived genes arise from such events. Rather, we have identified a significant number of domesticated TEs that share a promoter with a neighbouring gene in a head-to-head, or bidirectional arrangement. We have further examined the role of CpG rich 'inherently bidirectional' promoters in the insertion of foreign elements in recently evolved genes and experimental systems.

\section{Results}

Domestication of protein-coding transposable elements in mammalian genomes

Recent studies have surveyed eukaryotic genomes for the presence of TEs that have been co-opted by their host genome [7-10]. In the human genome it has been estimated that approximately $0.1 \%$ of all protein-coding genes contain (at least) part of a TE, however little data are available related to the potential mechanisms leading to TE co-option [9]. We have utilised updated genome annotation and genome-wide expression data to expand this analysis to further examine the co-option status of inserted transposable elements by mammalian genomes (Tables 1 and 2 and Additional file 1 and 2). Since a high proportion of protein-coding genes in both humans and mouse contain small insertions from common TEs such as Alu and B1 type elements, we limited our survey to genes with protein coding regions of at least 30\% sequence length homology to a known TE (see Materials Methods).

Using these selection criteria, we identified a total of 63 transcribed protein-coding genes within the human genome derived from previously characterised TEs. These can be grouped into three main classes: DNA transposons, retrotransposons and endogenous retroviruses. The most abundant group are DNA transposons $(n=42)$ followed by retrotransposons $(n=19)$ and endogenous retroviruses $(\mathrm{n}=2)$ (Table 1 and Additional file 1). Of note, all of these elements contain intact open reading frames and evidence

Table I: Survey of human domesticated genes ${ }^{a}$

\begin{tabular}{|c|c|c|c|c|c|}
\hline Repeat family & Repeat type & Number & Chimeric & Bidirectional & Bidirectional with CpG island \\
\hline \multirow[t]{5}{*}{ DNA transposons } & Tigger & 13 & 2 & 8 & 8 \\
\hline & PiggyBac & 5 & 2 & I & I \\
\hline & THAP (P-element) & 12 & 2 & 6 & 6 \\
\hline & HSMAR (mariner) & 1 & 1 & 0 & 0 \\
\hline & Others & 11 & 2 & 3 & 3 \\
\hline \multirow[t]{3}{*}{ Retrotransposons } & Paraneoplastic antigens & 8 & 0 & 2 & 2 \\
\hline & Ty3/gypsy & 8 & 0 & 2 & 2 \\
\hline & Others & 3 & 1 & I & I \\
\hline Endogenous retroviruses & Syncytin & 2 & 0 & 0 & 0 \\
\hline Total & & 63 & $10(15.9 \%)$ & $23(36.5 \%)$ & 23 \\
\hline
\end{tabular}

a For a more detailed survey of domesticated TEs in the human genome see Additional file I 
Table 2: Survey of mouse domesticated genes ${ }^{\mathrm{a}}$

\begin{tabular}{llcccc}
\hline Repeat family & Repeat type & Number & Chimeric & Bidirectional & Bidirectinal with CpG island \\
\hline \multirow{2}{*}{ DNA transposons } & Tigger & 8 & 2 & 2 & 2 \\
& PiggyBac & 2 & 1 & 0 & 0 \\
& THAP (P-element) & 7 & 2 & 1 & 1 \\
\multirow{3}{*}{ Retrotransposons } & Others & 6 & 2 & 3 & 3 \\
& Paraneoplastic antigens & 7 & 0 & 1 & 2 \\
\multirow{2}{*}{ Endogenous retroviruses } & Ty3/gypsy & 8 & 0 & 2 & 1 \\
& Others & 3 & 0 & 0 & 2 \\
\hline \multirow{2}{*}{ Total } & Others & 2 & 0 & 2 & 2 \\
\hline
\end{tabular}

a For a more detailed survey of domesticated TEs in the mouse genome see Additional file 2

of transcriptional activity, suggesting production of functional proteins. (Additional files 1 and 2). Fewer TEderived genes were identified in the mouse genome (45 compared with 63). However, a similar distribution of TE classes was observed for mouse with 23 DNA transposons, 18 retrotransposons and 4 endogenous retroviruses.

\section{A link between TE domestication and bidirectional promoters?}

TE insertion into downstream regions (exons or introns) of annotated genes to create a chimeric gene has previously been reported (Figure 1 and Additional file 1 and 2) $[9,11]$. Of the 63 potential domesticated TE genes we have identified in the human genome, only $10(15.9 \%)$ are likely to have arisen in this manner. Thus, $84 \%$ of the domesticated genes show an alternative mechanism of insertion. Further analysis of the genomic landscape associated with this subclass revealed a disproportionate number that appear to share a common promoter arranged in an antisense orientation (ie. bidirectional pair).

To test the frequency of this arrangement we compared the incidence of TE-associated bidirectionality with the human genome average of bidirectional gene pairs. Exact estimates of the number of bidirectional genes in the human and mouse genomes vary, between $9.8 \%(4,226$ of 42,887 transcription units; [12]) and $11 \%$ of human genes $(2,700$ of 24,000 genes; [13]), and $8.9 \%$ of mouse transcriptional units $(3,276$ of 36,606$)$ exist as part of a bidirectional pair, with transcription start sites less than 1 $\mathrm{kb}$ apart $[12,13]$. Using similar criteria we observed that 23 of the $63(36.5 \%)$ co-opted TE genes exist as part of a bidirectional pair in humans which is significantly greater than the genome average of such an arrangement; $\chi^{2}=$ 39.3, $\mathrm{p}<0.0001$ (Table 1 and Additional file 1). The mouse genome also contains a significantly higher proportion of co-opted genes in bidirectional pairs (12 of 45, $26.7 \%)$ when compared with the genome average $\left(\chi^{2}=\right.$
17.5, p < 0.0001) (Table 2 and Additional file 2). In order to test whether the $30 \%$ TE similarity cut off used in our analysis preferentially identified genes with a reduced cDNA length relative to the genomic average, we calculated the mean cDNA length of bidirectional gene pairs relative to the genome-wide average. No significant difference between the two data sets was observed with a genome-wide mean cDNA length of 2465 bp (2130 bp median) [13] and a mean cDNA length of bidirectional pairs of $2352 \mathrm{bp}$ (2086 bp median).

Between 77\% [13] and 94\% [12] of bidirectional promoters in the human genome contain a classical CpG island, defined as a stretch of DNA over $200 \mathrm{bp}$, with a G + C content of over $50 \%$, and an observed/expected ratio of CG dinucleotides of over 0.6 [14]. This appears to be a specific feature of mammalian genomes [15]. The corresponding proportion for unidirectional promoters in the human genome is between $38 \%$ [13] and 60\% [12] depending on whether annotated genes or transcriptional units are used as a reference. $\mathrm{CpG}$ island length of bidirectional promoters appears significantly longer than unidirectional promoters [12]. However $\mathrm{CpG}$ island size is not strictly a determinant of bidirectionality status [16]. In human and mouse genomes we found that all cases of bidirectional promoters associated with a domesticated TE contain a CpG island that shows some overlap of the first exons of both the host and domesticated TE genes (Table 1 and 2, and Additional files 1 and 2).

\section{Inherent bidirectionality and open chromatin of CPG islands - a chink in the defensive armour}

Most CpG islands within the human genome exist in an unmethylated state and are associated with constitutively active genes $[17,18]$. Such a state is invariably associated with an open chromatin structure that is anticipated to render the underlying DNA accessible to chromatin-associated proteins involved in the transcriptional regulation. We postulated that the majority of promoters containing 

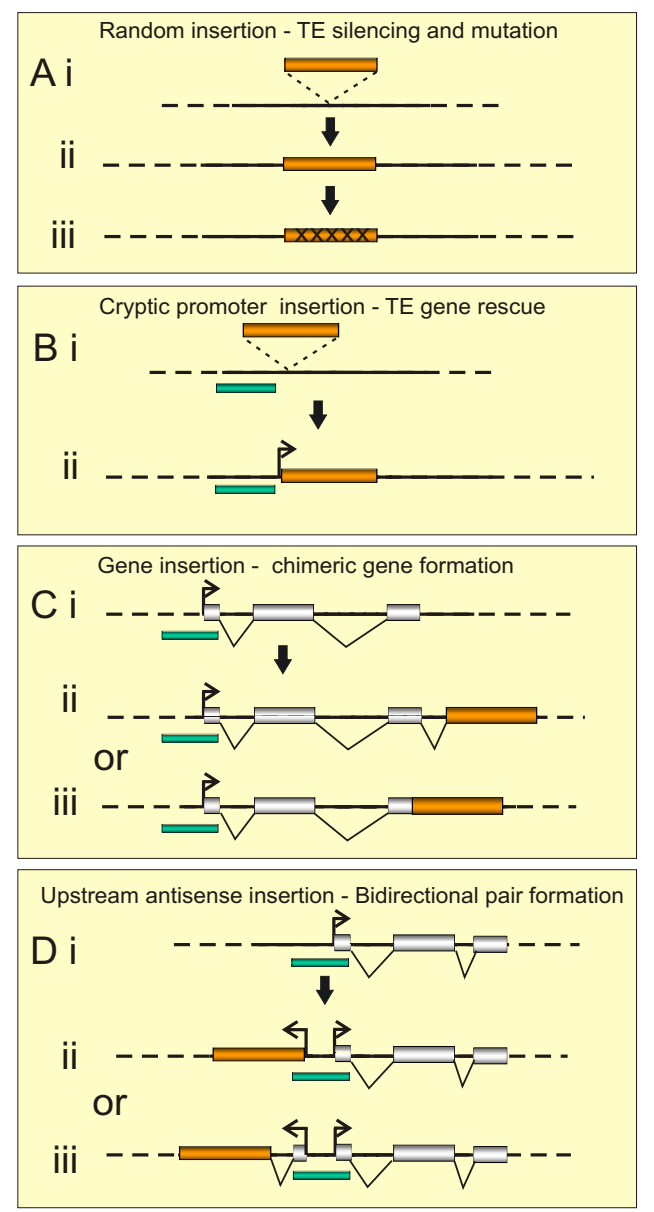

\section{Figure I}

Molecular models for transposable element cooption and domestication. (A) Transposable elements often insert into intergenic or intronic regions but are generally transcriptionally silenced through epigenetic modification. Over time mutations accumulate due to a lack of selective pressure to maintain sequences intact. If the TE contains sequences that drive transcription it may remain active if insertion takes place in a genomic location that favours expression over TE repression and silencing. (B) TE may insert into a region of the genome with inherent transcriptional activity or in close proximity to a cryptic CpG rich promoter (green box) that may result in TE avoiding epigenetic silencing to remain active. (C) Less frequently, TEs may insert downstream of the transcription start site of a host gene (i) either into an intron, or (ii) exon (iii) with the potential to create novel chimeric genes and/or proteins. (D) Insertion of a TE upstream of a host gene in an antisense direction. (i) Pre-insertion genomic environment displaying a typical multi-exon unidirectional gene. (ii) Incoming TE may insert within I kb of a CpG island gene promoter with transcription initiating within the TE itself. (iii) Alternatively the TE may insert further away from host promoter but will form a novel gene with a new upstream exon and an intervening intron that is spliced during mRNA formation.
CPG islands within mammalian genomes show inherent bidirectional capacity which, coupled with their open chromatin state, renders them ideal target sites for the integration and subsequent transcription of TEs arranged in an antisense direction to the endogenous partner gene. We anticipated that the constitutive transcriptional state of the promoter region of the founding member of the pair has the additional effect of protecting the incoming parasitic DNA element from the endogenous gene silencing machinery that would normally render such insertions inactive and prevent their expression following integration.

To test whether CpG islands associated with unidirectional transcription units have inherent properties permissive for bidirectional transcription, we investigated the nature of trapped loci that have been characterised as part of the International Gene Trap Consortium (IGTC) in mouse embryonic stem cell lines [19]. We screened the database for the presence of anti-sense transcripts which overlapped or were upstream of exon 1 of an annotated gene. These were aligned for position and direction within the mouse genome. We selected anti-sense gene-trap tags according to the following selection criteria; overlapping with exon 1 or the predicted promoter, and within $2 \mathrm{~kb}$ upstream of the annotated transcription start site or CpG island. Sequence tags located downstream of the annotated transcription start sites and known bidirectional genes were excluded from analysis.

From a total of 7592 Ensembl genes trapped by the IGTC as of 8-May-2006 we identified $11(0.145 \%)$ annotated genes or transcriptional units (TUs) associated with antisense trap-tags (Table 3 and Additional file 3). Of these, seven contain more than one tag in the anti-sense direction, and two loci are tagged with more than one type of gene-trap vector. Similar to the bi-directionally co-opted human and mouse TEs, all of the genes associated with anti-sense trap tags have an associated CpG island. Further evidence in support of antibiotic resistance gene cassette rescue by anti-sense transcription is the splicing of the resulting anti-sense transcript tags or rare ESTs, as observed for the Daxx, Kif2a and Uba52 genes. Cap analysis gene expression (CAGE) tags indicative of potential anti-sense transcription start sites [20] were also found at most sites of bidirectional gene-trap insertions (Table 3).

Further data supporting an inherent bidirectionality of 'unidirectional' gene promoters in the human genome has also been described [13]. Of 56 random nonbidirectional promoters assayed for transcriptional activity in vitro, 52\% were active in both directions. In contrast, $90 \%$ of 258 assayed bidirectional promoters were active in both directions. Data from this study also suggests that bidirectional promoters share a common DNA region necessary for 
Table 3: Anti-sense rescue of gene trap cassettes in mouse ES cells ${ }^{a}$

\begin{tabular}{lccrr}
\hline Gene & Anti-sense gene trap tags & CpG island & CAGE tag sense $^{\mathbf{a}}$ & CAGE tag anti-sense $^{\mathbf{b}}$ \\
\hline 2610209A20Rik & 1 & yes & 44 & 1 \\
Atp5b & 1 & yes & 3843 & 7 \\
Daxx & 2 & yes & 427 & 19 \\
Fnbp4 & 12 & yes & 1170 & 7 \\
Kif2a & 2 & yes & 335 & 7 \\
KpnbI & 4 & yes & 166 & 81 \\
BC09639I & 2 & yes & 367 & 36 \\
Ppmlb & 3 & yes & 36 & 1 \\
Rab30 & 1 & yes & 511 & 24 \\
Top3b & 1 & yes & 1743 & 0 \\
Uba52 & 7 & yes & 4 \\
\hline
\end{tabular}

a See Additional file 3 for more details.

b Cap analysis of gene expression (CAGE) tags were scored within I kb of the consensus start site of transcription. Sense and anti-sense CAGE tags are orientated with respect to the listed trapped gene.

transcription in both directions and therefore exist as inseparable functional units [13].

In combination, these data demonstrate the inherent capacity for bidirectional transcription of $\mathrm{CpG}$ islandassociated promoters in mammals, adding support to our hypothesis that such sites are suitable for the opportunistic insertion and expression of foreign TE transcriptional units into mammalian genomes.

\section{TIGDI, a recently domesticated DNA-based transposable} element

TIGD1 is an example of a recent co-opted transposable element present in human and chimp genomes as part of a bidirectional gene pair. DNA sequence analysis reveals that TIGD1 retains the conserved catalytic DDE/D core but lacks one of the terminal inverted repeats necessary for transposition (data not shown) [21]. To further delineate the extent of conservation and estimate the time of acquisition of this element in evolution, we searched available genomic sequence datasets or performed PCR analysis on the intergenic junction region between the TIGD1 and EIF4E2 genes in a panel of primate and other mammalian genomic DNAs. TIGD1 was detected in all primate species including human, Pan troglodytes (chimp), Pan paniscus (bonobo chimp), Gorilla gorilla, Pongo pygmaeus (orangutan), Macaca mulata (rhesus monkey), Macaca nemestrina (pigtailed macaque), Saguinus labiatus (tamarin), Ateles geoffroyi (spider monkey), Otolemur garnetti (small-eared galago) and Lemur catta (ring-tailed lemur) (Figure 2 and Additional file 4). However, TIGD1 is absent in other mammalian groups examined including the tree shrew, Tupaia belangeri (Scandentia) and the little brown bat, Myotis lucifugus (Chiroptera), the next closet representative orders to primates. Therefore TIGD1 domestication occurred concurrently with the divergence of the primate lineage between 67 to 98 million years ago [22].

\section{Murine bidirectional endogenous retroviruses}

The Friend virus susceptibility gene (Fv1) confers resistance against the murine leukaemia virus in mice $[23,24]$. This endogenous retrovirus appears to have been a recent co-option event, since it is not present in the rat genome. It shares homology with the gag domain of other endogenous mammalian retroviruses, especially the mouse MuERV-L (Figure 3). Fv1 exists in a bidirectional pairing with the D4Wsu114e gene (transcription start sites $<250$ bp apart). Although endogenous retroviruses are generally flanked by 5' and 3' LTR sequences (5'LTR sequence conferring promoter activity), no such promoter LTR elements appear to have contributed to the insertion and retention of this endogenous retroviral element.

Another mouse-specific endogenous retrovirus of unknown function that has recently been co-opted is the 4632419I22Rik gene. This exists as part of a bidirectional pair with Brip1, (transcription start sites 170 bp apart). PCR amplification of a panel of rodent species revealed the presence of this gene in all Mus species including; $M$. musculus, M. musculus castaneus, M. spretus, M. famulus, $M$. caroli, M. pahari, and M. saxicola (Figure 4 and Additional file 5). These results suggest that 4632419I22Rik appeared with the Mus lineage, after the divergence of the Rattus sensu lato and Praomyini (containing Mastomys) clades [25]. Similar to the Fv1 insertion, 4632419I22Rik does not include a 5'LTR sequence (Figure 3).

\section{Retroposed genes}

Retroposed genes usually arise from the reverse transcription of a host cell RNA with subsequent integration of the intronless cDNA into the host genome at a new location, usually in the absence of any upstream regulatory elements. This process is thought to be mediated by the by the reverse transcriptase enzyme of the L1 family of retrotransposons [1]. To investigate the degree to which transcribed retroposed genes are inserted and maintained 

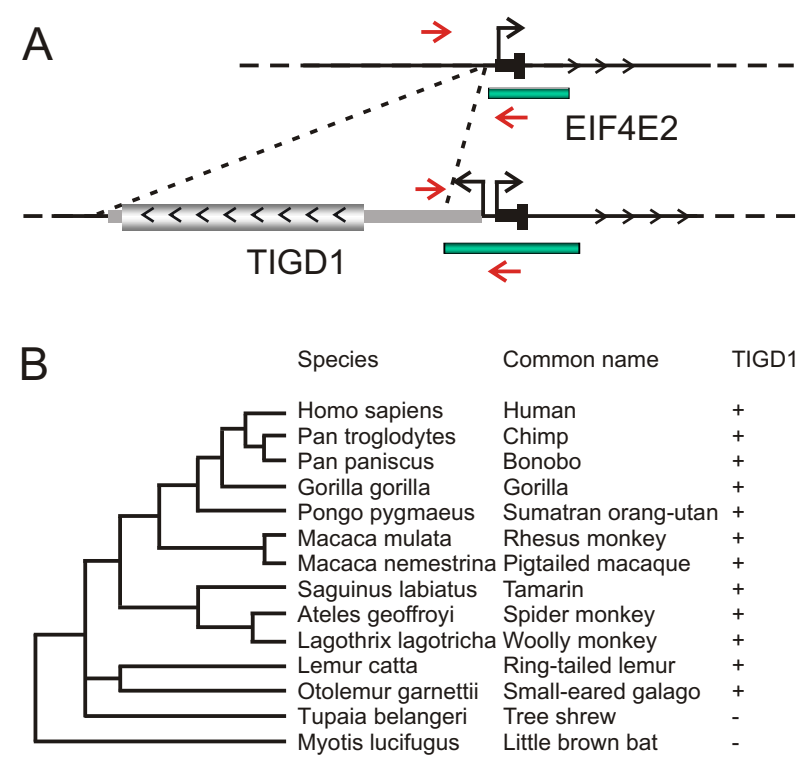

Figure 2

Formation of the TIGDI gene in primates. (A) Schematic representation pre- and post- insertion of the TIGDI ancestral TE with locations of amplification primers (red arrows) used to screen for the presence of the TIGDI gene in mammalian species and CpG island (green bars). Non-coding exons are denoted by narrow boxes and coding exons by larger shaded boxes with the direction of transcription indicated by arrowheads. (B) Molecular phylogenetic analysis of the domestication of TIGDI in primates and a summary of TIGDI bioinformatic or PCR screening assay. (+) denotes the presence of the TIGDI gene and $(-)$ denotes the absence of this gene within specific species. Phylogenetic tree of primate lineage displayed.

through inherent bidirectional activity of CpG island promoters, we examined the genomic location of the top 20 transcribed retroposed genes in the human genome [26] (Additional file 6). Of these, six (30\%) were found to be bidirectional (transcription start site within $1 \mathrm{~kb}$ of an annotated gene) and associated with a CpG island. When we extended our analysis to include the top 50 transcribed retrogenes, no additional instances of bidirectionality were observed suggesting a possible link between bidirectionality and transcriptional activity.

\section{Genomic environment - pre and post insertion}

To examine the genomic landscape before and after gene insertion we chose a group of recently domesticated genes that exist as part of novel bidirectional pairings with a common CpG island promoter. This list comprised two primate-specific genes (TIGD1 and PGBD4) and two mouse-specific genes ( $F v 1$ and 4632419I22Rik). Each ancestral gene promoter was anticipated to be unidirectional. However, two of the four genes display some
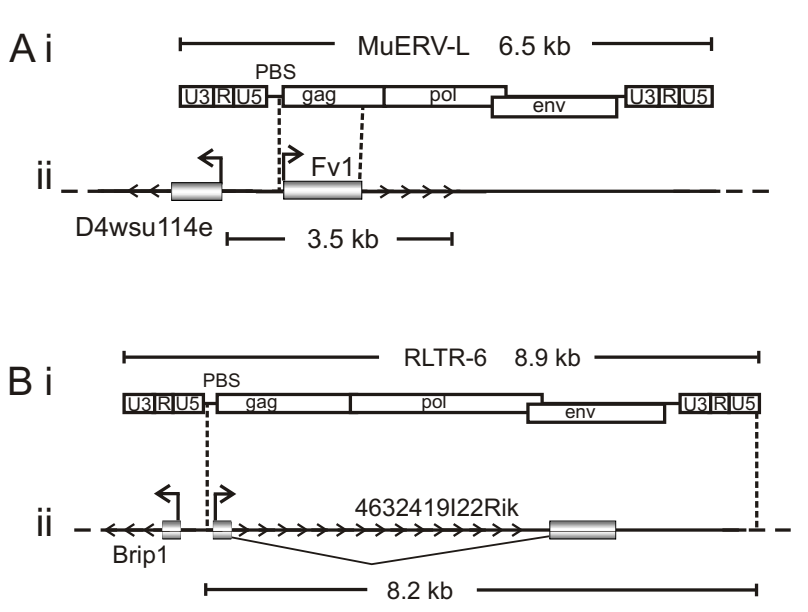

Figure 3

Bidirectional insertion of mouse endogenous retroviruses to form 46324I 9122Rik and FvI genes. (Ai and Bi) Presumed parental endogenous retroviruses, MuERV-L and RLTR-6 with LTR domains (U3-R-U5), primer binding site (PBS) and open reading frames, gag, pol and env. (Aii and Bii) Structure of the domesticated 46324I9122Rik and Fvl genes showing regions of homology to presumed ancestral ERV (dashed lines). Exons are denoted by grey boxes and direction of transcription is indicated with arrow heads.

degree of bidirectional activity as evidenced by CAGE and/or EST tags in species lacking the TE insertion element (Table 4 and Figure 5). TE insertion and maintenance of coding capacity appears to be associated with an increase in CpG island length for the TIGD1-EIF4E2, 4632419I22Rik-Brip1 and Fv1-D4Wsu114e promoter regions, whereas the CpG island associated with PGBD42900064A13Rik/C15ORF24 remained unchanged following TE insertion (Table 4). Increasing size is associated with an increase in the number of CpG dinucleotides in the region closest to the newly co-opted gene. Interestingly, this is also associated with an increase in the ratio of antisense: sense (co-opted: host) CAGE tags associated with each CpG island region post TE insertion, suggesting an increasing capacity for antisense transcription or increasing transcript stability following TE insertion (Table 4 and Figure 5).

\section{Co-expression of human TE-bidirectional gene pairs}

To examine whether the human TE-derived bidirectional gene pairs exhibit coordinate expression we performed a correlation analysis using publicly available GNF Gene Expression Atlas 2 data on 79 human tissues [27]. A Pearson correlation coefficient was calculated for nine gene pairs where both were included on the array and at least one of which exhibited detectable expression levels following array analysis (Additional file 7). Of the pairs examined 56\% (5/9) showed a correlation coefficient of 


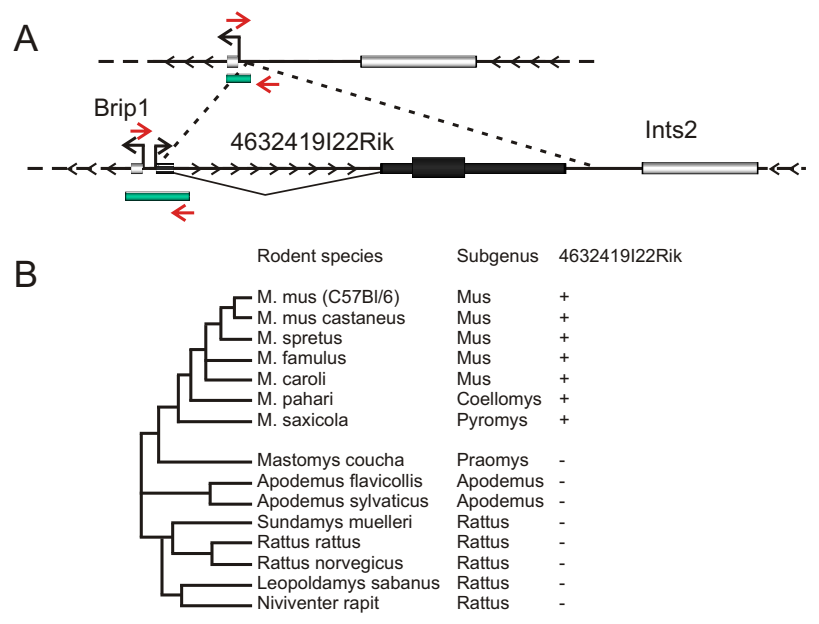

\section{Figure 4}

Formation of the 46324 I 9122 Rik gene in rodents. (A) Schematic representation pre and post insertion of a 46324I9122Rik ancestral TE with locations of amplification primers (red arrows) used to screen for the presence or absence of the 46324I9122Rik gene in mammalian species and $C_{p G}$ island (green bars). Non-coding exons of 46324 I9122Rik and neighbouring genes Bripl and Ints2 are denoted by narrow boxes and coding exons by larger shaded boxes with the direction of transcription indicated by arrowheads. (B) Molecular phylogenetic analysis of the domestication of $4632419122 \mathrm{Rik}$ in rodents and a summary of 4632419122 Rik screening assay. $(+)$ denotes the presence of the 4632419122 Rik gene and (-) denotes the absence of this gene within specific species. Phylogenetic tree of rodent lineage is displayed.

greater than 0.7 with $33 \%(3 / 9)$ showing a correlation value of over 0.8 . This supports previous data showing bidirectional gene pairs are more likely to be co-expressed when compared with random gene pairs [13].

\section{Discussion}

Most of the DNA that makes up complex genomes is parasitic in nature, derived from transposable elements acquired throughout evolution. Generally, these elements exist as neutrally evolving inactive DNA remnants (or fossils) silenced by the host genome over time. Relatively little attention has been paid to a minority of conserved TEs that retain protein coding capacity, and are expressed with varying degrees of cell-type specificities. Many of these are conserved across phylogeny, suggesting a degree of selective pressure over time that may be indicative of functional importance $[21,28]$.

The process of "domestication" of such transposable element-encoded proteins is just beginning to be appreciated and begins with a co-option (integration and expression) of a TE into the host genome. Numerous examples of such elements contributing to the acquisition of specific novel host cell functions have been described in a variety of organisms. Some of these examples include regulation of light signalling in Arabidopsis [29], mammalian neuronal development [30] and resistance to viruses in mice [23]. A recent study highlighting this phenomenon demonstrated that inactivation of a retrotransposon-like element in mice leads to embryonic lethality due to placental defects [31]. Thus, a complex evolutionary interplay appears to exist between genomic silencing of transposon elements to prevent their proliferation, and co-option of transposon-encoded proteins to provide novel cellular functions in higher eukaryotic genomes.

Previous studies have implicated downstream insertion of TEs into functional genes as a major mechanism for TE rescue (in the absence of an incoming promoter element), co-option and the formation of chimeric proteins of novel function. There are numerous examples of such insertions

Table 4: Transcriptional activity, pre and post transposable element insertion

\begin{tabular}{|c|c|c|c|c|c|c|}
\hline Species & Host Gene & CpG Island Length (bp) & CAGE tags & $\begin{array}{c}\text { Antisense } \\
\text { Co-opted gene }\end{array}$ & CAGE tags & CAGE tag ratio \\
\hline mouse & Eif4e2 & 566 & $174,205^{a}$ & - & $6,5^{a}$ & $0.034,0.024^{a}$ \\
\hline human & EIF4E2 & 806 & 173 & TIGDI & 51 & 0.29 \\
\hline mouse & 2900064A I3Rik & 469 & 1736 & - & 0 & 0 \\
\hline human & CI5ORF24 & 450 & 1509 & PGBD4 & 7 & 0.0046 \\
\hline human & IIP45 & 652 & 18 & - & 1 & 0.056 \\
\hline rat & D4Wsul I4e & 250 & $9 \mathrm{~b}$ & - & $0^{\mathrm{b}}$ & 0 \\
\hline mouse & D4Wsul I4e & 301 & 63 & $\mathrm{Fvl}$ & 19 & 0.3 \\
\hline human & BRIPI & 242 & 9 & - & 0 & 0 \\
\hline rat & Bripl & 211 & $6^{\mathrm{b}}$ & - & $0^{\mathrm{b}}$ & 0 \\
\hline mouse & Bripl & 856 & 129 & 46324I II22Rik & 312 & 2.4 \\
\hline
\end{tabular}

a Two transcriptional start sites that are approximately 400 bp apart

${ }^{b}$ No CAGE data available for rat therefore ESTs were used instead 

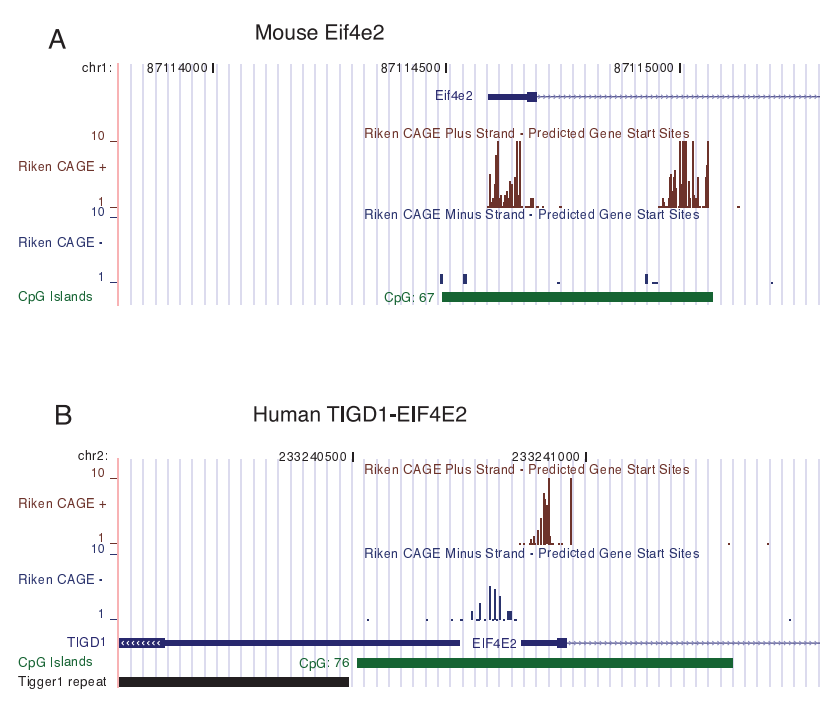

Figure 5

Transcriptional activity and $\mathrm{CpG}$ island genomic environment, pre and post insertion of TIGDI. (A) Promoter region of the mouse Eif4e 2 gene shows a predominantly unidirectional grouping of cap analysis gene expression (CAGE) tag transcription start sites in the plus direction (brown vertical bars) together with some detectable activity in the minus direction (blue vertical bars). (B) After the insertion of the TIGDI element in the primate lineage, the human TIGDI-EIF4E2 promoter region displays a lengthening of the $C_{P G}$ island and bidirectional transcription of the TIGDI and EIF4E2 genes, blue and brown vertical bars, respectively. The Riken CAGE transcription start sites are overlayed on a $\mathrm{I}, 500 \mathrm{bp}$ window for mouse and human genome assemblies $\mathrm{mm} 5$ and hg 17 , respectively.

in mammalian genomes. However, our analysis suggests that a more common mechanism of genomic TE cooption involves insertion of an incoming TE upstream of a host gene, oriented in an antisense direction, to form novel bidirectional gene pairs. Further, this appears to occur primarily at genes regulated by CpG-containing promoters, with expression and maintenance of TE integrity facilitated by the inherent bidirectional transcriptional capacity of $\mathrm{CpG}$ promoter regions.

\section{Permissive chromatin environment for insertion and transcription of transposable elements}

The majority of $\mathrm{CpG}$ islands within mammalian genomes exist in an unmethylated state and are associated with constitutively active genes $[17,18]$. This state is associated with an open chromatin structure that is thought to render the underlying DNA accessible to various chromatin associated proteins involved in the transcriptional process. We postulated that the majority of $\mathrm{CpG}$ islands within mammalian genomes show inherent bidirectional capacity, which coupled with their open chromatin state, make them ideal target sites for the integration and ectopic transcription of opportunistic parasitic transposable elements. In support of this supposition, our analysis of co-opted TEs and rescued gene-trap cassettes revealed that all such insertion events occurred within the vicinity of $\mathrm{CpG}$ island regulatory regions. Additional support comes from the use of a synthetic DNA transposon, Sleeping Beauty, as a mutagenesis tool in mammalian genomes. From over 1,000 insertion events mapped in human and mouse cell lines, a small but significant insertion bias towards the $5^{\prime}$ upstream regions of genes has been reported [32].

CAGE tag and other expression analyses have shown that CpG island promoters display a broad and complex distribution of transcription start sites when compared to nonCpG island promoters $[33,34]$ possibly contributing to the observed inherent bidirectional potential. Transcription factors like Sp1 can bind at multiple sites and protect the CpG island promoter from being silenced [35-37]. In contrast, when a TE inserts into a non-CpG island or inactive chromatin region, then it is usually silenced via the cis-spreading of silencing factors and/or the homologydependent RNAi silencing machinery thereby protecting the host genome from the mutagenic potential of subsequent TE insertions $[4,5]$.

\section{Conclusion}

In this study we have described a major molecular mechanism for co-option of transposable elements by host mammalian genomes involving the antisense insertion upstream of inherently bidirectional promoters. This does not affect the integrity of the ancestral gene partner unlike the related gene fusion events that involve downstream DNA insertion into existing genes in the same orientation $[1,9]$. The net effect of the events we have identified is the acquisition of an additional (and often novel) gene, predicted to acquire a specific gene expression profile dependent of the nature of the associated CpG-containing promoter. Often, the resulting tissue specificity of expression from the incoming DNA element is correlated with its bidirectional partner gene. In many instances such genes carry out essential functions within host organisms and have therefore been 'domesticated' by the host during evolution $[7,8]$. This appears to be frequently associated with the divergence of specific mammalian lineages during evolution. Further studies on the role of co-opted TEs are warranted to determine the exact roles each plays in mammalian development and speciation.

\section{Methods \\ Selection criteria for the inclusion of co-opted protein- coding genes in this study}

Human and mouse genes were selected as co-opted using the following criteria. 1) At least $30 \%$ of the coding region 
was derived from a known transposable element. Transposable element similarity was determined using PSIBLAST with up to four iterations of the NCBI non-redundant protein database, or direct searches of the Repbase repetitive DNA database [38]. To avoid false positives, simple repeat sequences were filtered out. 2) The gene showed evidence of expression by EST/mRNA cDNA clones from human and mouse UniGene datasets, build \#216 and \#176, respectively. In addition, we used the Novartis human and mouse tissue gene expression atlas data sets to confirm the cloned EST/mRNA results [27]. 3) We limited the classification to protein-coding genes which were manually annotated and reviewed by RefSeq or UniProt database curators. Mean and median cDNA lengths calculated for bidirectional gene pairs and genome wide genes were retrieved from the UCSC Genome Browser website [39] using the GenBank accession numbers provided in a previous study of bidirectional gene pairs [13]. The whole genome survey of coopted TEs was limited to human and mouse genomes since both organisms have extensive expression datasets and almost completely sequenced genomes.

\section{Definition of a bidirectional gene}

A gene pair was classified as bidirectional if the consensus transcription start sites were within $1 \mathrm{~kb}$ of each other. The major start site peak position was extracted from the CAGE data sets for human hg17 and mouse mm5 genome assemblies [40].

\section{Detecting the presence or absence of co-opted genes in vertebrate genomes}

To determine that a co-opted gene was either present or absent in genomes other than human we used the chained alignments available at the UCSC Genome Brower (human hg18 and mouse mm9 assemblies) [39]. The coopted gene plus at least $5 \mathrm{~kb}$ of flanking sequence were used to assess whether it was present or absent in the compared genome. Furthermore, the flanking $5 \mathrm{~kb}$ sequence was searched against the genome sequence datasets to ensure that the TE insertion had not arisen due to segmental duplication.

\section{Statistical analyses}

Chi-squared analysis was used to test the association of co-opted genes with bidirectional promoters. Pearsons correlation coefficient was used to determine the level of correlation between human bidirectional gene pairs from expression data in 79 human tissues [27]. Expression was called as present $(\mathrm{P})$ or absent (A) according to the Affymetrix array analysis tools.

\section{CPG island analysis}

CpG islands were detected according to the criteria set by the UCSC genome browser which is a modification of
[14]. CpG islands were predicted by searching the sequence one base at a time, scoring each dinucleotide (+17 for CG and -1 for others) and identifying maximally scoring segments. Each segment was then evaluated for the following criteria: GC content of $50 \%$ or greater, length greater than $200 \mathrm{bp}$, ratio greater than 0.6 of observed number of CG dinucleotides to the expected number on the basis of the number of Gs and Cs in the region of interest.

\section{PCR and sequence analyses}

PCR amplification across the junction regions of the TIGD1 and 4632419I22Rik co-opted genes was performed using HotStar Taq polymerase (Qiagen) using the manufacturer's instructions. Primate genomic DNA panel and the rodent DNA panels were obtained from, Coriell Cell Repositories and Dr Francois Catzeflis, respectively. Primer pairs for the presence or absence of the TIGD1 gene were; TIGD1-f1 5'-CAGGGCTGCCACAAACCC-3' and EIF4-r1 5'-GTCGAACTTGTTGTTCATCCTC-3' or CHRNG-f1 5'-AGCA(A/G)GTTCATTT(T/C)ATTTACTCC$3^{\prime}$ and EIF4-r1, respectively. Primer pairs for the presence or absence of the 4632419I22Rik gene were; Brip1-f 5'AATTCGCGCCTCCCGC-3' and 463Rik-r 5'-GCGTCCTCCAGGACTCTTCG-3', and Brip1-f and Ints2-r 5'GGAAATTGTACTTCTTGGCAAGG-3', respectively. PCR product integrity was verified by either direct DNA sequencing, or ligating into pGEM-Teasy vector (Promega) followed by sequencing.

\section{List of abbreviations}

TE: transposable element; IGTC: International Gene Trap Consortium; TU: transcriptional unit; CAGE: cap analysis gene expression; EST: expressed sequence tag; LTR: long terminal repeat.

\section{Authors' contributions}

PK and RS designed and performed research, analysed and wrote the paper.

\section{Additional material}

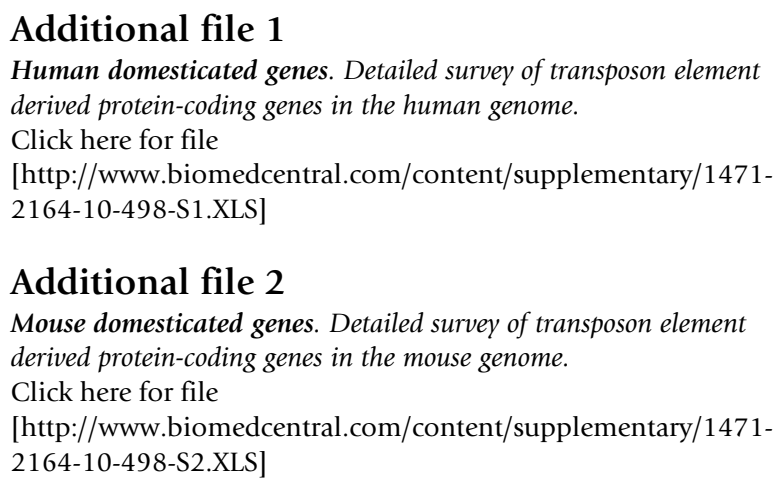




\section{Additional file 3 \\ Anti-sense rescue of gene trap cassettes in mouse ES cell lines. This dataset provides evidence that $C p G$ island promoters have the capacity of rescuing gene trap cassettes in a bidirectional manner. \\ Click here for file \\ [http://www.biomedcentral.com/content/supplementary/1471- 2164-10-498-S3.XLS] \\ Additional file 4 \\ Multiple sequence alignment of TIGD1-EIF4E2 junction in primates. The multiple sequence alignment displays the 5 ' insertion site of the TIGD1 gene in the primate lineage. \\ Click here for file \\ [http://www.biomedcentral.com/content/supplementary/1471- 2164-10-498-S4.DOC] \\ Additional file 5 \\ Multiple sequence alignments of Brip1-4632419I22Rik and Brip1- \\ Ints2 junctions. The data show the 5' insertion site of the \\ 4632419I22Rik gene before and after molecular domestication. \\ Click here for file \\ [http://www.biomedcentral.com/content/supplementary/1471- \\ 2164-10-498-S5.DOC] \\ Additional file 6 \\ Bidirectional retroposed genes. A survey of the top 20 transcribed retro- posed genes in the human genome showed that six of them were orientated in a bidirectional manner. \\ Click here for file \\ [http://www.biomedcentral.com/content/supplementary/1471- 2164-10-498-S6.XLS] \\ Additional file 7 \\ Expression microarray correlation analysis of human TE-derived bidi- rectional gene pairs. The data show that a high proportion of human TE- derived bidirectional gene pairs exhibit concordant tissue expression with the neighbouring gene. \\ Click here for file \\ [http://www.biomedcentral.com/content/supplementary/1471- 2164-10-498-S7.XLS]}

\section{Acknowledgements}

We would like to thank Dr Francois Catzeflis for providing phylogenetic expertise and the rodent DNA panel, Dr Nick Wong, Dr Katrina Bell and Ms Lavinia Gordon for bioinformatic assistance, Prof John Carlin for statistical advice and Dr Damien Hudson for helpful comments on the manuscript. This work was supported by an Australian Research Council Discovery Project grant, DP0773186. PK is an RD Wright Fellow of the Australian National Health and Medical Research Council.

\section{References}

I. Long M, Betran E, Thornton K, Wang W: The origin of new genes: glimpses from the young and old. Nat Rev Genet 2003, 4(I I):865-875.

2. Doolittle WF, Sapienza C: Selfish genes, the phenotype paradigm and genome evolution. Nature 1980, 284(5757):60 I-603.

3. Orgel LE, Crick FH, Sapienza C: Selfish DNA. Nature 1980, 288(5792): $645-646$.

4. Lippman Z, Martienssen R: The role of RNA interference in heterochromatic silencing. Nature 2004, 43 I (7006):364-370.
5. O'Donnell KA, Boeke JD: Mighty Piwis defend the germline against genome intruders. Cell 2007, I 29(I):37-44.

6. Miller WJ, McDonald JF, Nouaud D, Anxolabehere D: Molecular domestication--more than a sporadic episode in evolution. Genetica 1999, 107(1-3): 197-207.

7. Zdobnov EM, Campillos M, Harrington ED, Torrents D, Bork P: Protein coding potential of retroviruses and other transposable elements in vertebrate genomes. Nucleic Acids Res 2005, 33(3):946-954.

8. Volff JN: Turning junk into gold: domestication of transposable elements and the creation of new genes in eukaryotes. Bioessays 2006, 28(9):913-922.

9. Gotea $\mathrm{V}$, Makalowski W: Do transposable elements really contribute to proteomes? Trends Genet 2006, 22(5):260-267.

10. Piriyapongsa J, Rutledge MT, Patel S, Borodovsky M, Jordan IK: Evaluating the protein coding potential of exonized transposable element sequences. Biol Direct 2007, 2:31.

II. Lee SH, Oshige M, Durant ST, Rasila KK, Williamson EA, Ramsey H, Kwan L, Nickoloff JA, Hromas R: The SET domain protein Metnase mediates foreign DNA integration and links integration to nonhomologous end-joining repair. Proc Natl Acad Sci USA 2005, 102(50): $18075-18080$.

12. Engstrom PG, Suzuki H, Ninomiya N, Akalin A, Sessa L, Lavorgna G Brozzi A, Luzi L, Tan SL, Yang L, et al.: Complex Loci in human and mouse genomes. PLoS Genet 2006, 2(4):e47.

13. Trinklein ND, Aldred SF, Hartman SJ, Schroeder DI, Otillar RP, Myers RM: An abundance of bidirectional promoters in the human genome. Genome Res 2004, I4(I):62-66.

14. Gardiner-Garden M, Frommer M: CpG islands in vertebrate genomes. J Mol Biol 1987, 196(2):26 I-282.

15. Koyanagi KO, Hagiwara M, Itoh T, Gojobori T, Imanishi T: Comparative genomics of bidirectional gene pairs and its implications for the evolution of a transcriptional regulation system. Gene 2005, 353(2): 169-176.

16. Adachi N, Lieber MR: Bidirectional gene organization: a common architectural feature of the human genome. Cell 2002, 109(7):807-809.

17. Bird AP: CpG-rich islands and the function of DNA methylation. Nature 1986, 32 I(6067):209-2I3.

18. Larsen F, Gundersen G, Lopez R, Prydz H: CpG islands as gene markers in the human genome. Genomics 1992, I3(4): 1095-I I07.

19. International Gene Trap Consortium [http://www.gene trap.org/index.html]

20. Katayama S, Tomaru Y, Kasukawa T, Waki K, Nakanishi M, Nakamura M, Nishida H, Yap CC, Suzuki M, Kawai J, et al: Antisense transcription in the mammalian transcriptome. Science 2005 , 309(5740): $1564-1566$.

21. Casola C, Hucks D, Feschotte C: Convergent domestication of pogo-like transposases into centromere-binding proteins in fission yeast and mammals. Mol Biol Evol 2008, 25(I):29-4I.

22. Steiper ME, Young NM: Primate molecular divergence dates. Mol Phylogenet Evol 2006, 4 I(2):384-394.

23. Best S, Le Tissier P, Towers G, Stoye JP: Positional cloning of the mouse retrovirus restriction gene FvI. Nature 1996, 382(6594):826-829.

24. Qi CF, Bonhomme F, Buckler-White A, Buckler C, Orth A, Lander MR, Chattopadhyay SK, Morse HC: Molecular phylogeny of FvI. Mamm Genome 1998, 9(12): 1049-1055.

25. Suzuki H, Shimada T, Terashima M, Tsuchiya K, Aplin K: Temporal, spatial, and ecological modes of evolution of Eurasian Mus based on mitochondrial and nuclear gene sequences. Mol Phylogenet Evol 2004, 33(3):626-646.

26. Vinckenbosch N, Dupanloup I, Kaessmann H: Evolutionary fate of retroposed gene copies in the human genome. Proc Natl Acad Sci USA 2006, 103(9):3220-3225

27. Su Al, Wiltshire T, Batalov S, Lapp H, Ching KA, Block D, Zhang J, Soden R, Hayakawa M, Kreiman G, et al: A gene atlas of the mouse and human protein-encoding transcriptomes. Proc Natl Acad Sci USA 2004, I 0 I(16):6062-6067.

28. Malik HS, Henikoff S: Positive selection of Iris, a retroviral envelope-derived host gene in Drosophila melanogaster. PLoS Genet 2005, I(4):e44.

29. Lin R, Ding L, Casola C, Ripoll DR, Feschotte C, Wang H: Transposase-derived transcription factors regulate light signaling in Arabidopsis. Science 2007, 3 18(5854): $1302-1305$. 
30. Toth M, Grimsby J, Buzsaki G, Donovan GP: Epileptic seizures caused by inactivation of a novel gene, jerky, related to centromere binding protein-B in transgenic mice. Nat Genet 1995, I I(I):7I-75

31. Ono R, Nakamura K, Inoue K, Naruse M, Usami T, Wakisaka-Saito N, Hino T, Suzuki-Migishima R, Ogonuki N, Miki H, et al.: Deletion of Peg I0, an imprinted gene acquired from a retrotransposon, causes early embryonic lethality. Nat Genet 2006, 38(I): $101-106$.

32. Yant SR, Wu X, Huang Y, Garrison B, Burgess SM, Kay MA: Highresolution genome-wide mapping of transposon integration in mammals. Mol Cell Biol 2005, 25(6):2085-2094.

33. Smale ST, Kadonaga JT: The RNA polymerase II core promoter. Annu Rev Biochem 2003, 72:449-479.

34. Carninci P, Sandelin A, Lenhard B, Katayama S, Shimokawa K, Ponjavic J, Semple CA, Taylor MS, Engstrom PG, Frith MC, et al:: Genomewide analysis of mammalian promoter architecture and evolution. Nat Genet 2006, 38(6):626-635.

35. Brandeis M, Frank D, Keshet I, Siegfried Z, Mendelsohn M, Nemes A, Temper V, Razin A, Cedar H: SpI elements protect a CpG island from de novo methylation. Nature 1994, 37 I (6496):435-438.

36. Macleod D, Charlton J, Mullins J, Bird AP: SpI sites in the mouse aprt gene promoter are required to prevent methylation of the CpG island. Genes Dev 1994, 8(1 9):2282-2292.

37. Mummaneni $P$, Yates $P$, Simpson J, Rose J, Turker MS: The primary function of a redundant Sp I binding site in the mouse aprt gene promoter is to block epigenetic gene inactivation. Nucleic Acids Res 1998, 26(22):5163-5169.

38. Repbase: repetitive DNA database [http://www.girinst.org/rep base/index.html]

39. UCSC Genome Browser [http://www.genome.ucsc.edu/]

40. Fantom3: Functional Annotation of the mouse - 3 [http:// fantom3.gsc.riken.jp/index.html]

Publish with Bio Med Central and every scientist can read your work free of charge

"BioMed Central will be the most significant development for disseminating the results of biomedical research in our lifetime. "

Sir Paul Nurse, Cancer Research UK

Your research papers will be:

- available free of charge to the entire biomedical community

- peer reviewed and published immediately upon acceptance

- cited in PubMed and archived on PubMed Central

- yours - you keep the copyright

Submit your manuscript here:

http://www.biomedcentral.com/info/publishing_adv.asp
BiolMedcentral 